\title{
COMPRESSIVE SENSING OF LIGHT FIELDS
}

\author{
S. Derin Babacan ${ }^{1}$, Reto Ansorge ${ }^{1}$, Martin Luessi ${ }^{1}$, Rafael Molina ${ }^{2}$, Aggelos K. Katsaggelos ${ }^{1}$ \\ ${ }^{1}$ EECS Department \\ Northwestern University, Evanston, IL USA \\ ${ }^{2}$ Departamento de Ciencias \\ de la Computación e I.A. \\ sdb@northwestern.edu, reto.ansorge@u.northwestern.edu, Universidad de Granada, Granada, Spain \\ m-luessi@northwestern.edu, aggk@eecs.northwestern.edu \\ rms@decsai.ugr.es
}

\begin{abstract}
We propose a novel camera design for light field image acquisition using compressive sensing. By utilizing a randomly coded nonrefractive mask in front of the aperture, incoherent measurements of the light passing through different regions are encoded in the captured images. A novel reconstruction algorithm is proposed to recover the original light field image from these acquisitions. Using the principles of compressive sensing, we demonstrate that light field images with high angular dimension can be captured with only a few acquisitions. Moreover, the proposed design provides images with high spatial resolution and signal-to-noise-ratio (SNR), and therefore does not suffer from limitations common to existing light-field camera designs. Experimental results demonstrate the efficiency of the proposed system.
\end{abstract}

Index Terms - Compressive sensing, light-fields, coded aperture imaging, computational photography, Bayesian methods.

\section{INTRODUCTION}

Recent advances in computational photography provided effective solutions to a number of photographic problems, and also resulted in novel methods to acquire and process images. Light-field cameras are one of the most widely used class of computational cameras. The light-field expresses the radiance density function on the camera sensor, or the light energy of all rays in 3D space passing through the camera. For instance, a four-dimensional (4D) discrete light field image $\mathbf{x}(i, k, m, n)$ with spatial dimensions $i, k$ and angular dimensions $m, n$ contains images of a scene from a variety of angles, which provide information about the 3D structure of the scene. The angular data provides means to work directly on the light-rays instead of pixels, allowing one to produce many views of the scene, or perform many photographic tasks after the acquisition is made. This provides a clear advantage for light-field imaging over traditional photography and makes many novel applications possible.

A number of light-field camera designs is proposed in the literature. The most common ones are plenoptic cameras utilizing microlens arrays [1,2], multi-camera [3] or multi-lens systems [4], and mask-based designs $[5,6]$. Many of these designs suffer from the spatio-angular tradeoff [4], that is, one cannot obtain light-field images with both high spatial- and high angular resolution. Recently, a programmable aperture camera is proposed [7] which uses a coded aperture to multiplex angular images into a single 2D image which is then decoded using linear estimation. This design captures images with both high spatial and angular resolution, but the number of acquisitions is equal to the number of angular dimensions. Therefore,

This work was supported in part by the Comisión Nacional de Ciencia y Tecnología under contract TIC2007-65533 and the Spanish research programme Consolider Ingenio 2010: MIPRCV (CSD2007-00018). obtaining a light-field image with a high angular resolution is not practical.

Compressive sensing (CS) $[8,9]$, on the other hand, has recently become very popular due to its interesting theoretical nature and wide area of applications. The theory of compressive sensing dictates that a signal can be recovered very accurately from a much smaller number of measurements than required by traditional methods, provided that it is compressible or sparse in some basis, i.e., only a few basis coefficients contain the major part of the signal energy. Besides sparsity, compressive sensing makes use of incoherent measurements and nonlinear reconstruction, and has led to many interesting theoretical results and novel applications (see, for instance, [10]).

In this paper, we present a novel application of compressive sensing, namely, a new camera design for light-field image acquisition. We build our design on ideas from coded aperture imaging, computational photography and compressive sensing. By exploiting the fact that different regions of the aperture correspond to images of the scene from different angles, we incorporate a compressively coded mask in front of the aperture to obtain incoherent measurements of the incident light-field. These measurements are then decoded using a novel reconstruction algorithm to recover the original light-field image. We exploit the highly sparse nature of the lightfield images to obtain accurate reconstructions with only a few measurements compared to the high angular dimension of the light-field image.

This paper is organized as follows: In Sec. 2 we present the proposed acquisition method to obtain incoherent measurements of the light field image. The Bayesian model utilized for the reconstruction is described in Sec. 3, and the reconstruction algorithm is developed in Sec. 4. We demonstrate the efficiency of the proposed system with experimental results in Sec. 5 and conclusions are drawn in Sec. 6.

\section{LIGHT FIELD ACQUISITION}

The basic principle of the proposed light-field acquisition system is the fact that different regions of the aperture capture images of the scene from different angles. In other words, the main camera lens can be interpreted as an array of multiple virtual lenses (or cameras). This concept is illustrated in Fig. 1(a)-(c), where only certain parts of the aperture are left open to acquire images exhibiting vertical and horizontal parallax. By separately opening one region of the aperture and blocking light in the others, the complete light-field with an angular dimension of $N$ can be captured with $N$ exposures. However, obtaining the light-field image in this fashion has two disadvantages: First, due to the very small amount of light at each exposure, the captured angular images have very low signal-to-noise ratios (SNR). Second, a high number of acquisitions has to be made in order to obtain high angular resolution. The programmable aperture camera 


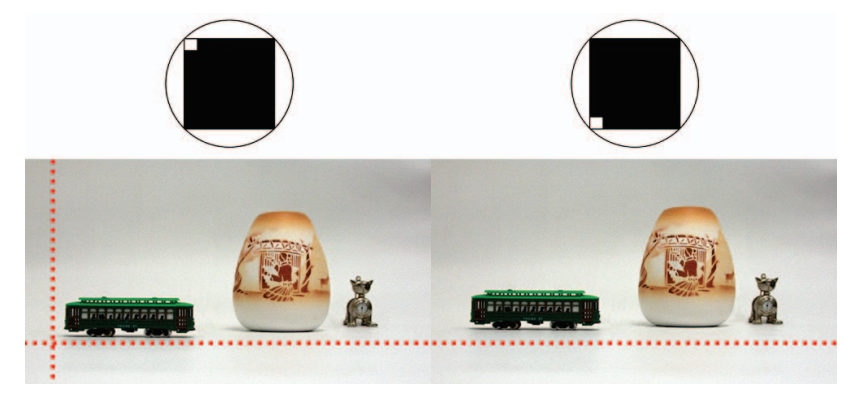

(a)

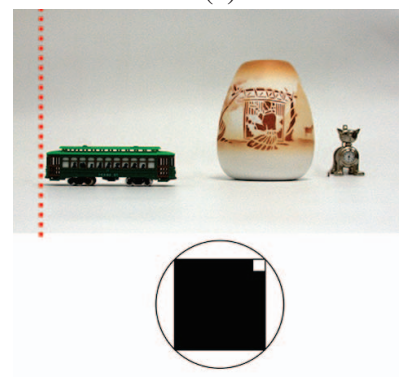

(c)

(d)

Fig. 1. The basic principle of utilizing a coded aperture to obtain light field images. The angular images are shown in (a), (b) and (c) when only corner blocks of the aperture are left open. Both horizontal and vertical parallax can be observed between these images (Horizontal and vertical dashed lines are shown to clearly denote the vertical and horizontal parallax, respectively). Figure (d) shows a captured image with the randomly coded aperture used in the proposed compressive sensing light field camera. All images are from a synthetic light field image (see Sec. 5).

design in [7] addressed the first problem and increased the SNR in each captured image through multiplexing, but it still suffers from the second problem.

We address both of these issues by utilizing a randomly coded non-refractive mask in front of the aperture. Each image acquired in this fashion is a random linear combination (and therefore an incoherent measurement) of the angular images. An example image captured in this fashion is illustrated in Fig. 1(d), where the amount of light passing through regions of the aperture are randomly selected (shown at the bottom of Fig. 1(d)).

Let us assume that the aperture of the main camera lens is divided into $N$ blocks, with $N=N_{h} \times N_{v}$ where $N_{h}$ and $N_{v}$ represent the number of horizontal and vertical divisions. During each acquisition $i$, each block $j$ is assigned a weight $0 \leq a^{i j} \leq 1$ which controls the amount of light passing through this block. Therefore, $a^{i j}$ represents the transmittance of the block $j$, i.e., it is the fraction of incident light that passes through the block. As mentioned above, each block $j$ captures an angular image $\mathbf{x}^{j}$ in the light-field image, and therefore the acquired image $\mathbf{y}^{i}$ at the $i^{\text {th }}$ acquisition can be represented as a linear combination of the $N$ angular images as

$$
\mathbf{y}^{i}=\sum_{j=1}^{N} a^{i j} \mathbf{x}^{j}, i=1, \ldots, M
$$

Therefore, the measurements after $M$ acquisitions (with $M \leq N$ ) can be expressed in matrix form as

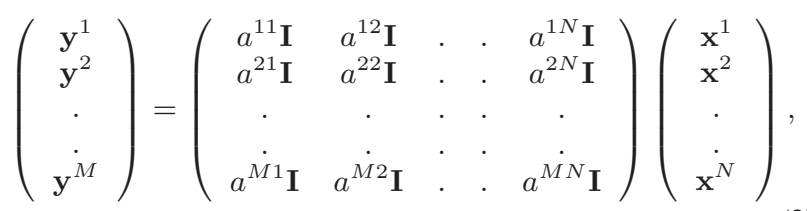

where $\mathbf{I}$ is the $P \times P$ identity matrix with $P$ the number of pixels in images $\mathbf{x}^{j}$ (and therefore, in $\mathbf{y}^{i}$ ). Taking the acquisition noise also into account, the final observation model can be expressed as

$$
\mathbf{y}=\mathbf{A x}+\mathbf{n} .
$$

It is clear that the light field acquisition system in (3) can be seen as a noisy incoherent measurement system, when the linear measurement matrix A satisfies certain properties dictated by the theory of compressive sensing [8]. The design of the measurement matrices for compressive sensing is an active area of research, and many of the existing designs can be utilized for the proposed aperture mask. In this work, the entries of $\mathbf{A}$ are drawn from random distributions. For instance, if the mask is limited to binary codes, scrambled Hadamard ensembles can be used to code the aperture [11]. A more general class of matrices can be utilized in cases where fractional values of the block transmittances are permitted.

Finally, it should be noted that since many (or possibly all) blocks are open in each exposure, each captured image has a high SNR due to the small amount of loss of light. In fact, the measurement matrices can be designed to optimize the amount of passing light while maintaining the random structure. Note that the traditional camera is a special case of the system in (3) with $M=1$ and $a^{11}=a^{12}=\ldots=a^{1 N}=1$, in which case the SNR is maximized, but the $3 \mathrm{D}$ structure is lost.

\section{BAYESIAN MODEL FOR RECONSTRUCTION}

In order to be able to reconstruct the angular images $\mathrm{x}^{1}, \mathrm{x}^{2}, \ldots, \mathrm{x}^{N}$ from the incoherent measurements $\mathbf{y}^{1}, \mathbf{y}^{2}, \ldots, \mathbf{y}^{M}$ and $\mathbf{A}$, we utilize a Bayesian framework by employing a conditional distribution $\mathrm{p}(\mathbf{y} \mid \mathbf{x}, \beta)$ for the observation model in (3) and a prior distribution $\mathrm{p}\left(\mathbf{x} \mid \boldsymbol{\alpha}_{\mathrm{TV}}, \boldsymbol{\alpha}_{c}\right)$ on the unknown light field image x. These distributions depend on the model parameters $\beta, \alpha_{\mathrm{TV}}$ and $\boldsymbol{\alpha}_{c}$, which are called hyperparameters and are also modeled within the proposed framework. We utilize the following factorization of the joint distribution $\mathrm{p}\left(\mathbf{y}, \mathbf{x}, \beta, \boldsymbol{\alpha}_{\mathrm{TV}}, \boldsymbol{\alpha}_{c}\right)$ of all unknown and observed quantities

$\mathrm{p}\left(\mathbf{y}, \mathbf{x}, \beta, \boldsymbol{\alpha}_{\mathrm{TV}}, \boldsymbol{\alpha}_{c}\right)=\mathrm{p}(\mathbf{y} \mid \mathbf{x}, \beta) \mathrm{p}\left(\mathbf{x} \mid \boldsymbol{\alpha}_{\mathrm{TV}}, \boldsymbol{\alpha}_{c}\right) \mathrm{p}(\beta) \mathrm{p}\left(\boldsymbol{\alpha}_{\mathrm{TV}}\right) \mathrm{p}\left(\boldsymbol{\alpha}_{c}\right)$

In this work, the hyperparameters $\beta, \boldsymbol{\alpha}_{\mathrm{TV}}$, and $\boldsymbol{\alpha}_{c}$ are modeled by uniform distributions. The specific models utilized for the rest of the distributions are presented in the following subsections.

\subsection{Observation (Noise) Model}

The observation noise is assumed to be independent and Gaussian with zero mean and variance equal to $\beta^{-1}$, that is, using (3),

$$
\mathrm{p}(\mathbf{y} \mid \mathbf{x}, \beta)=\mathcal{N}\left(\mathbf{y} \mid \mathbf{A x}, \beta^{-1}\right) .
$$

\subsection{Light-Field Image Model}

The choice of randomly programmed coded apertures makes the exact/approximate recovery of angular images possible through the use of sparsity inherent in light field images. There are multiple sources 
of sparsity within light field images that can be exploited. The first one is sparsity within each angular image. It is already well known that $2 \mathrm{D}$ images can be very accurately represented by only a few coefficients of a sparsifying transform, such as wavelet transforms or total-variation (TV) functions on the image. In the case of light-field images, there is another fundamental source of sparsity, that is, the angular images are very closely related to each other. Specifically, each angular image can be accurately estimated from another one using dense warping (or correspondence) fields.

Based on the above, we utilize the following factorization of the prior distribution $\ln \mathrm{p}\left(\mathbf{x} \mid \boldsymbol{\alpha}_{\mathrm{TV}}, \boldsymbol{\alpha}_{c}\right)=\ln \mathrm{p}\left(\mathbf{x} \mid \alpha_{\mathrm{TV}}\right)+\ln \mathrm{p}\left(\mathbf{x} \mid \alpha_{c}\right)+$ $\ln C\left(\alpha_{\mathrm{TV}}, \alpha_{c}\right)$, where $\mathrm{p}\left(\mathbf{x} \mid \alpha_{\mathrm{TV}}\right)$ is the TV image prior employed on each angular image separately, $\mathrm{p}\left(\mathbf{x} \mid \alpha_{c}\right)$ is the prior modeling the sparsity arising from the strong dependency between angular images and $C\left(\alpha_{\mathrm{TV}}, \alpha_{c}\right)$ is a function of the unknown hyperparameters needed for the image prior model to integrate to one.

Specifically, $\mathrm{p}\left(\mathbf{x} \mid \boldsymbol{\alpha}_{\mathrm{TV}}\right)$ can be expressed as

$$
\mathrm{p}\left(\mathbf{x} \mid \boldsymbol{\alpha}_{\mathrm{TV}}\right) \propto \prod_{i=1}^{N}\left(\alpha_{\mathrm{TV}}^{i}\right)^{P / 2} \exp \left[-\frac{1}{2} \alpha_{\mathrm{TV}}^{i} \mathrm{TV}\left(\mathbf{x}^{i}\right)\right],
$$

where

$$
\operatorname{TV}\left(\mathbf{x}^{i}\right)=\sum_{k} \sqrt{\left(\Delta_{k}^{h}\left(\mathbf{x}^{i}\right)\right)^{2}+\left(\Delta_{k}^{v}\left(\mathbf{x}^{i}\right)\right)^{2}},
$$

where $\Delta_{k}^{h}$ and $\Delta_{k}^{v}$ correspond to, respectively, horizontal and vertical first order differences, at pixel $k$, that is, $\Delta_{k}^{h}\left(\mathbf{x}^{i}\right)=\left(\mathbf{x}^{i}\right)_{k}-$ $\left(\mathbf{x}^{i}\right)_{l(k)}$ and $\Delta_{k}^{v}\left(\mathbf{x}^{i}\right)=\left(\mathbf{x}^{i}\right)_{k}-\left(\mathbf{x}^{i}\right)_{a(k)}$, where $l(k)$ and $a(k)$ denote the nearest neighbors of pixel $k$, to the left and above, respectively.

Next, let us denote by $\mathbf{M}^{k j}$ the dense warping field between the images $\mathbf{x}^{k}$ and $\mathbf{x}^{j}$, that is, $\mathbf{x}^{k} \approx \mathbf{M}^{k j} \mathbf{x}^{j}$. This dependency of each angular image on another is very strong and can be exploited while modeling $\mathbf{x}$. Therefore, we utilize the following cross-image prior between the angular images

$$
\mathrm{p}\left(\mathbf{x} \mid \boldsymbol{\alpha}_{c}\right) \propto \exp \left(\sum_{k=1}^{N} \sum_{j \in \mathcal{N}(k)}-\frac{\alpha_{c}^{k j}}{2}\left\|\mathbf{x}^{k}-\mathbf{M}^{k j} \mathbf{x}^{j}\right\|^{2}\right),
$$

where $\mathcal{N}(k)$ denotes the angular images in the neighborhood of $\mathbf{x}^{k}$. We define the neighborhood as images one angular dimension apart to reduce the computational complexity and to use accurate warping fields. The cross-image prior in (8) can be written in matrix-vector form as

$$
\mathrm{p}\left(\mathbf{x} \mid \boldsymbol{\alpha}_{c}\right) \propto \exp \left(-\frac{1}{2} \mathbf{x}^{T} \Pi \mathbf{x}\right),
$$

where the matrix $\Pi$ is a sparse $N P \times N P$ matrix constructed by $N \times N$ blocks of size $P \times P$. Specifically, it is given by

$$
\Pi=\left(\begin{array}{ccccc}
\Pi_{11} & \Pi_{12} & \cdot & \cdot & \Pi_{1 N} \\
\Pi_{21} & \Pi_{22} & \cdot & \cdot & \Pi_{2 N} \\
\cdot & \cdot & \cdot & \cdot & \cdot \\
\cdot & \cdot & \cdot & \cdot & \cdot \\
\Pi_{N 1} & \Pi_{N 2} & \cdot & \cdot & \Pi_{N N}
\end{array}\right)
$$

The $P \times P$ blocks $\Pi_{k j}$ can be found from (8) as

$$
\Pi_{k j}= \begin{cases}\sum_{s \in \mathcal{N}(k)} \alpha_{c}^{k s} \mathbf{I}+\alpha_{c}^{s k}\left(\mathbf{M}^{s k}\right)^{T} \mathbf{M}^{s k} & \text { if } j=k \\ -\alpha_{c}^{k j} \mathbf{M}^{k j}-\alpha_{c}^{j k}\left(\mathbf{M}^{j k}\right)^{T} & \text { if } j \neq k, j \in \mathcal{N}(k) \\ 0 & \text { else }\end{cases}
$$

It is clear that incorporating the cross-image prior requires knowledge of the dense warping fields $\mathbf{M}^{j k}$, which cannot be directly obtained from the compressive measurements. In this work, we overcome this problem by acquiring two additional images from two opposite sides of the aperture. These images exhibit full horizontal and vertical parallax, and a dense registration algorithm based on graph-cuts is utilized to obtain the warping field [12]. Due to the uniform partitioning of the aperture, this warping field can be used to obtain approximate intermediate warping fields between all angular images.

\section{RECONSTRUCTION ALGORITHM}

Utilizing the joint distribution in (4), we obtain the maximum a posteriori estimate of the light field image $\mathbf{x}$ as

$$
\begin{aligned}
\hat{\mathbf{x}} & =\Sigma_{\mathbf{x}} \beta \mathbf{A}^{T} \mathbf{y} \\
\Sigma_{\mathbf{x}}^{-1} & =\operatorname{diag}\left(\alpha_{\mathrm{TV}}^{i}\left(\Delta^{h}\right)^{t} \mathbf{W}_{\mathrm{TV}}^{i}\left(\Delta^{h}\right)+\alpha_{\mathrm{TV}}^{i}\left(\Delta^{v}\right)^{t} \mathbf{W}_{\mathrm{TV}}^{i}\left(\Delta^{v}\right)\right)+\Pi
\end{aligned}
$$

where the first matrix term in (12) is a $N P \times N P$ block diagonal matrix created by $P \times P$ blocks $\alpha_{\mathrm{TV}}^{i}\left(\Delta^{h}\right)^{t} \mathbf{W}_{\mathrm{TV}}^{i}\left(\Delta^{h}\right)+$ $\alpha_{\mathrm{TV}}^{i}\left(\Delta^{v}\right)^{t} \mathbf{W}_{\mathrm{TV}}^{i}\left(\Delta^{v}\right)$. The matrices $\mathbf{W}_{\mathrm{TV}}^{i}$ are calculated by

$$
\mathbf{W}_{\mathrm{TV}}^{i}=\operatorname{diag}\left(\frac{1}{\sqrt{\left(\mathbf{w}_{\mathrm{TV}}^{i}\right)_{k}}}\right), k=1, \ldots P
$$

where

$$
\left(\mathbf{w}_{\mathrm{TV}}^{i}\right)_{k}=\left(\Delta_{k}^{h}\left(\hat{\mathbf{x}}^{i}\right)\right)^{2}+\left(\Delta_{k}^{v}\left(\hat{\mathbf{x}}^{i}\right)\right)^{2} .
$$

In this work we use the following estimates of the hyperparameters

$$
\begin{aligned}
\beta & =\frac{N P}{\|\mathbf{y}-\mathbf{A} \hat{\mathbf{x}}\|^{2}}, \\
\alpha_{\mathrm{TV}}^{i} & =\frac{\frac{1}{2} P}{\sum_{k} \sqrt{\left(\mathbf{w}_{\mathrm{TV}}^{i}\right)_{k}}}, \\
\alpha_{c}^{i j} & =\frac{P}{\left\|\hat{\mathbf{x}}^{i}-\mathbf{M}^{i j} \hat{\mathbf{x}}^{j}\right\|^{2}} .
\end{aligned}
$$

In calculating (16) we have used the quadratic bound provided by the majorization-minimization approach proposed in [13].

Finally, the algorithm iterates among estimating the light field image using (11), the spatial adaptivity vectors using (14), and the hyperparameters using (15)-(17) until convergence.

\section{EXPERIMENTAL RESULTS}

For the results reported in this paper, we generated a synthetic lightfield image shown in Fig. (1) with known warping fields. The light field image has a spatial resolution of $250 \times 125$ and an angular resolution of $7 \times 7$. As the measurement matrix $\mathbf{A}$ we chose the uniform spherical ensemble, that is, its entries are drawn from a uniform distribution and are between 0 and 1 . Since the mean of this distribution is 0.5 , using this measurement matrix, the expected amount of light passing through the aperture in each acquisition is half of the maximum possible. Finally, we add zero-mean Gaussian noise with variance 0.1 to the measurements to obtain the final observations.

We vary the number of acquired images $M$ from 1 to 49 and apply the proposed reconstruction algorithm using the incoherent observations to obtain estimates of the original light-field image. Each 


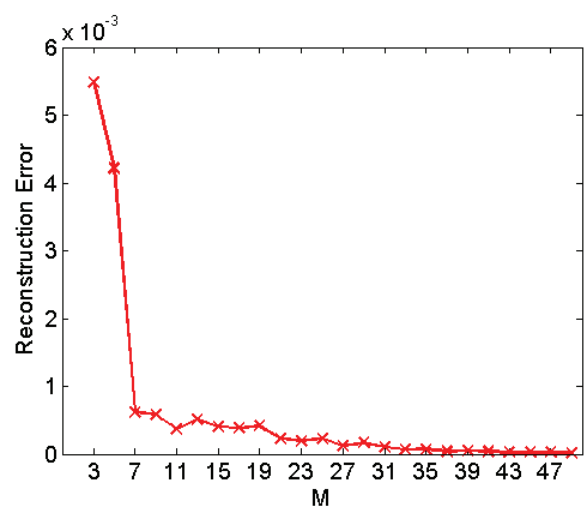

Fig. 2. Number of measurements $M$ vs relative reconstruction error (average over 50 runs).

experiment is repeated 50 times and the average is reported. The reconstruction error is calculated according to $\|\hat{\mathbf{x}}-\mathbf{x}\|_{2}^{2} /\|\mathbf{x}\|_{2}^{2}$, where $\mathbf{x}$ and $\hat{\mathbf{x}}$ are the original and estimated images, respectively.

Average reconstruction errors over 50 runs are shown in Fig. (2). It is clear that very accurate reconstructions can be obtained using very few measurements. The minimum reconstruction error that can be achieved is around $0.5 \times 10^{-5}$ with 49 measurements, due to the presence of observation noise. The proposed algorithm provides average reconstruction errors of around $1 \times 10^{-4}$ and $1.5 \times 10^{-5}$ from 11 and 21 measurements, respectively. In fact, an average error of $6 \times 10^{-4}$ is already obtained with only 7 measurements. Examples of reconstructed images using 11 and 21 measurements are shown in Fig. 3(b) and Fig. 3(c), respectively. Note that the reconstructed images are nearly indistinguishable from the original image, which is shown in Fig. 3(a). It can be observed that using the proposed design the number of acquisitions can be significantly reduced (by a factor between $1 / 7$ to $1 / 4$ ). Furthermore, the reduction in the number of acquisitions is expected to be much higher with larger light-field images, due to the increased level of sparsity.

The proposed light-field camera design is also implemented using an SLR camera and a liquid crystal array as the spatial light modulator. We obtained promising real-image results which will be reported in the future.

\section{CONCLUSIONS}

In this paper, we proposed a novel application of compressive sensing to a novel camera design to acquire 4D light-field images. We have shown that incoherent measurements of the angular images can be collected by using a randomly coded mask in front of the aperture of a traditional camera. These measurements are then used to reconstruct the original light field image. We developed a reconstruction algorithm which exploits the high degree of sparsity inherent in the light field images, and have shown that the complete light field image can be reconstructed using only a few acquisitions. Moreover, the captured images have high signal-to-noise ratios due to small amount of loss of light. The proposed design provides high spatial and angular resolution light field images, and does not suffer from limitations of many existing light field images. Finally, the proposed design can be implemented by simple modifications of traditional cameras.

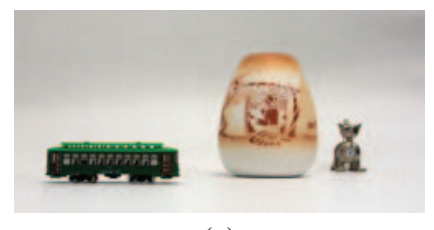

(a)

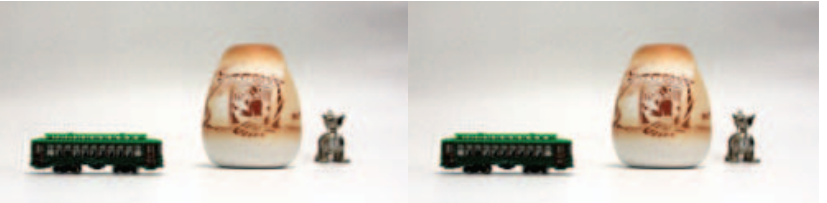

(b)

(c)

Fig. 3. Reconstruction examples. (a) Original angular image, reconstructed images from (b) 11 measurements (relative reconstruction error $=3.4 \times 10^{-4}$ ) and (c) 21 measurements (relative reconstruction error $=1.4 \times 10^{-5}$ ).

\section{REFERENCES}

[1] T. Adelson and J. Wang, "Single lens stereo with a plenoptic camera," IEEE Trans. Pattern Anal. Machine Intell., vol. 14, no. 2, pp. 99-106, Feb 1992.

[2] R. Ng, M. Levoy, M. Brdif, G. Duval, M. Horowitz, and P. Hanrahan, "Light field photography with a hand-held plenoptic camera," Stanford Tech. Rep., 2005.

[3] B. Wilburn, N. Joshi, V. Vaish, E. Talvala, E. Antunez, A. Barth, A. Adams, M. Levoy, and M. Horowitz, "High performance imaging using large camera arrays," in ACM Trans. Graph., 2005.

[4] T. Georgiev, K. C. Zheng, B. Curless, D. Salesin, S. Nayar, and C. Intwala, "Spatio-angular resolution tradeoffs in integral photography," in EGSR, june 2006, pp. 263-272.

[5] A. Veeraraghavan, R. Raskar, A. Agrawal, A. Mohan, and J. Tumblin, "Dappled photography: Mask enhanced cameras for heterodyned light fields and coded aperture refocusing," ACM Trans. Graph., vol. 26, no. 3, pp. 69:1-69:12, Jul. 2007.

[6] T. Georgiev, C. Intwala, S. D. Babacan, and A. Lumsdaine, "Unified frequency domain analysis of lightfield cameras," in ECCV, Marseille, France, December 2008.

[7] C.-K. Liang, T.-H. Lin, B.-Y. Wong, C. Liu, and H. H. Chen, "Programmable aperture photography: multiplexed light field acquisition,” ACM Trans. Graph., pp. 1-10, 2008.

[8] E. Candes, J. Romberg, and T. Tao, "Robust uncertainty principles: exact signal reconstruction from highly incomplete frequency information," IEEE Trans. Inform. Theory, vol. 52, no. 2, pp. 489-509, Feb. 2006.

[9] D. L. Donoho, "Compressed sensing," IEEE Trans. Inform. Theory, vol. 52, no. 4, pp. 1289-1306, 2006.

[10] R. Baraniuk, "Compressive sensing," IEEE Signal Processing Magazine, vol. 24, no. 4, pp. 118-121, July 2007.

[11] L. Gan, T. Do, and T. Tran, "Fast compressive imaging using scrambled block Hadamard ensemble," in EUSIPCO 2008, Lausanne, Switzerland, August 2008.

[12] Y. Boykov, O. Veksler, and R. Zabih, "Fast approximate energy minimization via graph cuts," IEEE Trans. Pattern Anal. Machine Intell., vol. 23, no. 11, pp. 1222-1239, Nov 2001.

[13] S. D. Babacan, R. Molina, and A. Katsaggelos, "Parameter estimation in TV image restoration using variational distribution approximation," IEEE Trans. Image Processing, vol. 17, no. 3, pp. 326-339, 2008. 